\title{
QUASI-ADDITIVE SOLUTIONS OF NONLINEAR DIFFERENTIAL EQUATIONS
}

\author{
A. S. JONES \\ (Received 29 January 1985)
}

\begin{abstract}
Some nonlinear second order ordinary differential equations have solutions which can be represented as sums of solutions of related equations. This paper classifies the equations for which this is possible and derives the corresponding forms.
\end{abstract}

1980 Mathematics subject classification (Amer. Math. Soc.): 34 A 25.

\section{Introduction}

In a recent paper, Whitham (1984) showed that solutions of the equation

$$
y^{\prime \prime}=6 B+(4-6 A) y-6 y^{2},
$$

which describe standing wave solutions of the Korteweg-de Vries equation, could be obtained in the form

$$
y=\sum_{-\infty}^{\infty} \operatorname{sech}^{2}(x-2 m \sigma),
$$

where $y=\operatorname{sech}^{2}\left(x-x_{0}\right)$ is the "soliton" solution of the related equation

$$
y^{\prime \prime}=4 y-6 y^{2} \text {. }
$$

Similar results, where standing wave solutions are constructed as infinite sums of solitons, are reported by Zaitsev (1983), who also considers two dimensional waves generated in this fashion.

(c) 1987 Australian Mathematical Society $0263-6115 / 87 \$ A 2.00+0.00$ 
In this paper we consider the general problem of constructing solutions of equations of the form $y^{\prime \prime}=p(y)$, where $p$ is a polynomial in $y$, as infinite sums of solutions of simpler, related equations. This includes the standing wave solutions by Zaitsev and Whitham, but also encompasses those real solutions which have singularities. The analysis is performed in the complex plane, and general results are derived with coefficients which are complex. The particular results which lead to real solutions for equations with real variables are then obtained by special choices of the parameters. The majority of the solutions obtained in this fashion have two distinct expansions as infinite sums of solutions of related equations, one using trigonometric functions and one involving hyperbolic functions. These represent the generalizations of the representations of standing wave solutions as superpositions of wave trains and as superpositions of solitons.

The most common of these expansions represents the solution not as a superposition of identical forms but as an alternating infinite sum of two forms both of which satisfy the same non-linear equation. Included in this category are a family of finitely periodic solutions of modified Korteweg-de Vries equations.

\section{Basic considerations}

Suppose that $y=f(z)$ is a meromorphic solution of a differential equation of the form $y^{\prime \prime}=p(y)$ for polynomial $p$ of degree $k$.

If $y$ has a pole of order $n$ at $z=a$, then $y^{\prime \prime}$ has a pole of order $n+2$ and $p(y)$ has a pole of order $k n$ at this point. Therefore, in order that $y$ be a solution, we must have $n+2=k n$ for integer $k, n$. The only solutions of this equation are $n=2, k=2$ and $n=1, k=3$. Hence we are restricted to polynomials of degrees 2 and 3, respectively. When $k=3, f(z)$ has only simple poles, and when $k=2, f(z)$ has only poles of order 2 .

Also, if $f$ has a pole of order 2, then we have

$$
\begin{aligned}
y & =\frac{A}{(z-\alpha)^{2}}+\frac{B}{(z-\alpha)}+g(z) ; \\
y^{\prime \prime} & =\frac{6 A}{(z-\alpha)^{4}}+\frac{2 B}{(z-\alpha)^{3}}+g^{\prime \prime}(z) ;
\end{aligned}
$$

and

$$
\frac{6}{A} y^{2}=\frac{6 A}{(z-\alpha)^{4}}+\frac{12 B}{(z-\alpha)^{3}}+O\left(\frac{1}{(z-\alpha)^{2}}\right) \text {. }
$$

Therefore, if $y^{\prime \prime}=a_{2} y^{2}+a_{1} y+a_{0}$, then $A=6 / a_{2}$ and $B=0$, i.e. the principal part of $f$ at every pole has the form $6 / a_{2}(z-\alpha)^{2}$. 
A similar analysis of $y^{\prime \prime}=a_{3} y^{3}+a_{2} y^{2}+a_{1} y+a_{0}$ shows, that at a simple pole the principal part of $y$ is $\pm A /(z-\alpha)$, where $A^{2}=2 / a_{3}$.

\section{Functions with second order poles}

In view of the preceding analyses, we take as our basic function having a second order pole the function $f(z)=A /(z-\alpha)^{2}$, which satisfies the second order equation $f^{\prime \prime}=6 f^{2} / A$, where the right hand side is itself the simplest possible quadratic polynomial.

If we now construct the new function $\Phi$, where $\Phi(z ; \omega)=\sum_{-\infty}^{\infty} f(z+n \omega)$, for arbitrary complex $\omega \neq 0$, we see that $\Phi$ is a periodic function with period $\omega$. The function $\Phi$ can be evaluated explicitly by using residue theory to give

$$
\begin{aligned}
\Phi & =-A \operatorname{Res}\left(\frac{\pi \cot \pi \zeta}{(\zeta \omega+z-\alpha)^{2}}\right) \quad \text { at } \zeta=\frac{\alpha-z}{\omega} \\
& =\frac{\pi^{2} A}{\omega^{2}} \operatorname{cosec}^{2}\left(\frac{\pi}{\omega}(z-\alpha)\right) .
\end{aligned}
$$

Direct computation shows that $\Phi$ is a solution of the equation

$$
\Phi^{\prime \prime}=\frac{6}{A} \Phi^{2}-\frac{4 \pi^{2}}{\omega^{2}} \Phi,
$$

so that, by inversion, we can express periodic solutions of this equation as infinite sums of solutions of the simpler equation which has only the leading term on the right hand side.

The functions $\operatorname{sech}^{2}\left(z-x_{0}\right)$ considered by Whitham correspond to $\Phi$ with $A=-1, \omega=\pi i$ and $\alpha=\frac{1}{2} \pi i+x_{0}$, and the corresponding sum

$$
\operatorname{sech}^{2}(z)=\sum_{-\infty}^{\infty} \frac{-1}{\left(z+\left(n+\frac{1}{2}\right) \pi i\right)^{2}}
$$

represents its normal partial fraction expansion.

Now if we choose a second complex period, independent of the first, we can construct the function

$$
F\left(z ; \omega_{1}, \omega_{2}\right)=\sum_{m=-\infty}^{\infty} \Phi\left(z+m \omega_{2} ; \omega_{1}\right) .
$$

This doubly periodic function can be expressed in terms of the function $\wp$ with periods $\omega_{1}, \omega_{2}$ by

$$
F=A\left(\wp(z-\alpha)+S_{2}\right)
$$


where

$$
\begin{aligned}
S_{2} & =\sum_{m=-\infty}^{\infty}\left(\sum_{n=-\infty}^{\infty} \frac{1}{\left(n \omega_{1}+m \omega_{1}\right)^{2}}\right) \\
(m, n) & \neq(0,0) \\
& =\frac{\pi^{2}}{\omega_{1}^{2}}\left(\frac{1}{3}+2 \sum_{m=1}^{\infty} \operatorname{cosec}^{2}\left(\frac{m \pi \omega_{2}}{\omega_{1}}\right)\right) \\
& =\frac{2 \eta_{1}}{\omega_{1}}, \quad \text { where } \eta_{1}=\zeta\left(\frac{1}{2} \omega_{1}\right) .
\end{aligned}
$$

Writing $\pi \omega_{2} / \omega_{1}=a+i b$, where $b \neq 0$, we have

$$
\left|\operatorname{cosec}^{2}\left(\frac{m \pi \omega_{2}}{\omega_{1}}\right)\right|=\frac{1}{\sin ^{2} m a+\sinh ^{2} m b} \leqslant \frac{1}{\sinh ^{2} m b},
$$

so that the single series expression for $S_{2}$ converges absolutely, and very rapidly since $\operatorname{cosech}^{2}(m b)<10^{-10}$ for $m b>12 \cdot 3$.

Changing the order of summation gives

$$
F\left(z ; \omega_{2}, \omega_{1}\right)=A\left(p(z-\alpha)+S_{2}^{*}\right)
$$

where

$$
S_{2}^{*}=\frac{\pi^{2}}{\omega_{2}^{2}}\left(\frac{1}{3}+2 \sum_{n=1}^{\infty} \operatorname{cosec}^{2}\left(\frac{m \pi \omega_{1}}{\omega_{1}}\right)\right)=\frac{2 \eta_{2}}{\omega_{2}}
$$

differs from $S_{2}$ due to the conditional convergence of the double sum.

The periods and the $\eta_{i}$ are connected by $\omega_{2} \eta_{1}-\omega_{1} \eta_{2}=\pi i$ when $\operatorname{Re}\left(\omega_{2} / i \omega_{1}\right)$ $>0$, so that $S_{2}-S_{2}^{*}=2 \pi i / \omega_{1} \omega_{2}$ in this case, and $F\left(z ; \omega_{1}, \omega_{2}\right)=F\left(z ; \omega_{2}, \omega_{1}\right)$ $+2 \pi i A / \omega_{1} \omega_{2}$. Since $\wp$ satisfies the equation

$$
\wp^{\prime \prime}=6 \wp^{2}-30 S_{4},
$$

where

$$
\begin{aligned}
S_{4} & =\sum^{\prime} \frac{1}{\left(m \omega_{2}+n \omega_{1}\right)^{4}} \\
& =\frac{\pi^{4}}{\omega_{1}^{4}}\left(\frac{1}{45}-\frac{4}{3} \sum_{1}^{\infty} \operatorname{cosec}^{2}\left(m n \frac{\omega_{2}}{\omega_{1}}\right)+2 \sum_{1}^{\infty} \operatorname{cosec}^{4}\left(\frac{m \pi \omega_{2}}{\omega_{1}}\right)\right),
\end{aligned}
$$

it follows that $F\left(z ; \omega_{1}, \omega_{2}\right)$ is a solution of the equation

$$
\begin{aligned}
F^{\prime \prime} & =\frac{6}{A} F^{2}-12 S_{2} F+A\left(6 S_{2}^{2}-30 S_{4}\right) \\
& =\frac{6}{A} F^{2}-\left(\frac{4 \pi^{2}}{\omega_{1}^{2}}\left(1+6 \sum\right)\right) F+B,
\end{aligned}
$$


which illustrates the structural relationship between this equation and that satisfied by $\Phi$.

The relationship between $F\left(z ; \omega_{1}, \omega_{2}\right)$ and $F\left(z ; \omega_{2}, \omega_{1}\right)$ implies that every doubly periodic solution admits two complementary expansions

$$
F=\sum_{-\infty}^{\infty} \Phi\left(z+m \omega_{2}, \omega_{1}\right)=\sum_{-\infty}^{\infty} \Phi\left(z+m \omega_{1} ; \omega_{2}\right)+\frac{2 \pi i A}{\omega_{1} \omega_{2}} .
$$

\section{Functions with first order poles}

A similar analysis holds for the case where the solution has simple poles.

In order to ensure convergence of the infinite sums it is necessary to take as the fundamental solution the function

$$
g(z)=A\left(\frac{1}{z-\alpha}-\frac{1}{z-\beta}\right),
$$

which satisfies the equation

$$
g^{\prime \prime}(z)=\frac{2}{A^{2}} g^{3}+\frac{6}{A(\alpha-\beta)} g^{2} .
$$

Proceeding as before, we construct

$$
\begin{aligned}
\psi(z ; \omega) & =\sum_{-\infty}^{\infty} g(z+n \omega) \\
& =\frac{2 \pi A}{\omega} \frac{\sin \theta}{\cos \theta-\cos (2 \pi z / \omega)},
\end{aligned}
$$

where $\theta=\pi(\alpha-\beta) / \omega$ and $Z=z-\frac{1}{2}(\alpha+\beta)$, and we see that $\psi$ satisfies the equation

$$
\psi^{\prime \prime}=\frac{2}{A^{2}} \psi^{3}+\frac{6}{A(\alpha-\beta)} \theta \cot \theta \psi^{2}-\frac{4 \pi^{2}}{\omega^{2}} \psi .
$$

The function sech $\zeta$ corresponds to $\alpha=-\beta=\pi i / 2, \omega=2 \pi i, A=-i$.

The double sum $G\left(z ; \omega_{1}, \omega_{2}\right)=\sum_{-\infty}^{\infty} \psi\left(z+m \omega_{2}, \omega_{1}\right)$ can also be represented in terms of the Weierstrass function.

Writing $H(Z)=G(z)$, where $Z=z-\frac{1}{2}(\alpha+\beta)$, we see that $H$ is an even function of $Z$ with simple poles at $A= \pm \frac{1}{2}(\alpha-\beta)= \pm r$, say.

Then $H(Z)-H(0)$ is doubly periodic with poles at $\pm r$ and a double zero at $Z=0$. Hence, if $\wp$ is the Weierstrass function with periods $\omega_{1}$ and $\omega_{2}$, then

$$
H(Z)=H(0)=\frac{A \wp^{\prime}(r)}{\wp(Z)-\wp(r)}
$$


and from this we find that $H$ (and hence $G$ ) satisfies the equation

$$
\begin{aligned}
H^{\prime \prime}= & \frac{2}{A^{2}} H^{3}+\left(\frac{3}{A} \frac{\wp^{\prime \prime}(r)}{\wp^{\prime}(r)}-\frac{6}{A^{2}} H(0)\right) H^{2} \\
& +\left(12 \wp(r)-\frac{6}{A} \frac{\wp^{\prime \prime}(r)}{\wp^{\prime}(r)} H \omega+\frac{6}{A^{2}} H(0)^{2}\right) H \\
& +\left(2 A \wp^{\prime}(r)-12 \wp(r) H(0)+\frac{3}{A} \frac{\wp^{\prime \prime}(r)}{\wp^{\prime}(r)} H^{2}(0)-\frac{2}{A^{2}} H^{3}(0)\right) .
\end{aligned}
$$

If we set

$$
y=\frac{H}{A}, \quad c=\frac{H(0)}{A} \quad \text { and } \quad f(t)=2 t^{3}+3 \frac{\wp^{\prime \prime}(r)}{\wp^{\prime}(r)} t^{2}+12 \wp(r) t+2 \wp^{\prime}(r),
$$

then this equation reduces to $y^{\prime \prime}=f(y-c)$.

The constant $c$ has the value

$$
\begin{aligned}
c & =\frac{2 \pi}{\omega_{1}}\left(-\cot \frac{r \pi}{\omega_{1}}+2 \sum_{1}^{\infty} \frac{\sin \left(2 r \pi / \omega_{1}\right)}{\cos \left(2 r \pi / \omega_{1}\right)-\cos \left(2 m \pi \omega_{2} / \omega_{1}\right)}\right) \\
& =\frac{2 r \eta_{1}}{\omega_{1}}-\zeta(r), \quad \text { where again } \eta_{1}=\zeta\left(\frac{\omega_{1}}{2}\right) .
\end{aligned}
$$

This series is also absolutely and rapidly convergent. As before,

$$
c^{*}=\frac{2 \pi}{\omega_{2}}\left(-\cot \frac{r \pi}{\omega_{2}}+2 \sum_{1}^{\infty} \frac{\sin \left(2 r \pi / \omega_{2}\right)}{\cos \left(2 r \pi / \omega_{2}\right)-\cos \left(2 m \pi \omega_{1} / \omega_{2}\right)}\right)
$$

differs from $c$, so that $H\left(Z ; \omega_{1}, \omega_{2}\right)-H\left(Z ; \omega_{2}, \omega_{1}\right)=A\left(c^{*}-c\right)=2 r A \pi i / \omega_{1} \omega_{2}$.

\section{Real equations with real solutions}

If we require functions which take real values when the independent variable is real, the choice of $\alpha$ and of the periods $\omega_{1}$ and $\omega_{2}$ is severely restricted.

The Weierstrass function $\varphi(z-\alpha)$ will be real for real $z$ provided its poles are symmetrically placed with respect to the $\operatorname{line} \operatorname{Im}(z-\alpha)=0$. This can occur in two ways.

Firstly we have $\omega_{1}$ real, $\omega_{2}=\frac{1}{2} \omega_{1}(1+i \lambda)$, so that the period parallelogram is a rhombus. Equivalently, we can take the periods as $\omega_{1}^{\prime \prime}=i \lambda \omega_{1^{\prime}}=2 \omega_{2}-\omega_{1^{\prime}}$ $\omega_{2}^{\prime \prime}=\omega_{2}$, which gives a different representation of the double periodic function.

In order that $\wp(t-\alpha)$ be real for real $t$, it is now necessary to take $\alpha$ real or $\alpha$ (real + period). Without loss of generality we take $\alpha_{1}=-\frac{1}{2} \omega_{1}$ when considering the first form of $\wp$ and $\alpha_{2}=\frac{1}{2} i \lambda \omega_{1}$ when considering the second. The function $\wp$ is unchanged by this, since $\alpha_{2}-\alpha_{1}$ is a period, although the representations alter. These choices of $\alpha$ make $\wp$ an even function of $t$. 
The first case occurs when the period parallelogram is rhomboidal. In this case $\wp$ has a simple representation in terms of the function $c n: \wp(u)=1 /\left(2-2 c_{n}(u)\right)$

$-\left(1+4 k^{2}\right) / 12$. Taking first $\omega=\omega_{1}, \alpha=-\frac{1}{2} \omega_{1}$, we have

and

$$
\Phi\left(t, \omega_{1}\right)=\frac{\pi^{2} A}{\omega_{1}^{2}} \sec ^{2}\left(\frac{\pi t}{\omega_{1}}\right)
$$

$$
\begin{aligned}
F_{1}\left(t ; \omega_{1}, \omega_{2}\right) & =\frac{\pi^{2} A}{\omega_{1}^{2}} \sum_{-\infty}^{\infty} \sec ^{2}\left(\frac{\pi t}{\omega_{1}}+\frac{m \pi}{2}+\frac{m \pi i \lambda}{2}\right) \\
& =\frac{\pi^{2} A}{\omega_{1}^{2}}\left(\sec ^{2} \frac{\pi t}{\omega_{1}}+4 \sum_{1}^{\infty} \frac{1+(-1)^{m} \cos \left(2 \pi t / \omega_{1}\right) \cosh (m \pi \lambda)}{\left(\cosh (m \pi \lambda)+(-1)^{m} \cos \left(2 \pi t / \omega_{1}\right)\right)^{2}}\right) .
\end{aligned}
$$

When we take $\omega=\omega_{1}^{\prime \prime}=i \lambda \omega_{1}, \alpha=\frac{1}{2} \lambda \omega_{1} i$, we obtain

$$
\Phi\left(t, \omega_{1}^{\prime \prime}\right)=-\frac{\pi^{2} A}{\lambda^{2} \omega_{1}^{2}} \operatorname{sech}^{2}\left(\frac{\pi t}{\lambda \omega_{1}}\right)
$$

and

$$
\begin{aligned}
& F_{1}\left(t ; \omega_{1}, \omega_{2}\right)=-\frac{\pi^{2} A}{\lambda^{2} \omega_{1}^{2}} \sum_{-\infty}^{\infty} \operatorname{sech}^{2}\left(\frac{\pi t}{\lambda \omega_{1}}+\frac{m \pi}{2 \lambda}+i \frac{m \pi}{2}\right) \\
& =-\frac{\pi^{2} A^{2}}{\lambda^{2} \omega_{1}^{2}}\left(\sum_{-\infty}^{\infty} \operatorname{sech}^{2}\left(\frac{\pi t}{\lambda \omega_{1}}+\frac{m \pi}{\lambda}\right)-\sum_{-\infty}^{\infty} \operatorname{cosech}^{2}\left(\frac{\pi t}{\lambda \omega_{1}}+\frac{(2 m-1) \pi}{2 \lambda}\right)\right) .
\end{aligned}
$$

In the first case,

$$
\begin{aligned}
S_{2} & =\frac{\pi^{2}}{\omega_{1}^{2}}\left(\frac{1}{3}+2 \sum_{1}^{\infty} \operatorname{sech}^{2}\left(\frac{(2 m-1)}{2} \pi \lambda\right)-2 \sum_{1}^{\infty} \operatorname{cosech}^{2}(-m \pi \lambda)\right) \\
& =\frac{\pi^{2}}{\lambda \omega_{1}^{2}} \mu_{2}(\lambda),
\end{aligned}
$$

while in the second case we have

$$
\begin{aligned}
S_{2}^{* *} & =-\frac{\pi^{2}}{\lambda^{2} \omega_{1}^{2}}\left(\frac{1}{3}+2 \sum_{1}^{\infty} \operatorname{sech}^{2}\left(\frac{(2 m-1) \pi}{2 \lambda}\right)-2 \sum_{1}^{\infty} \operatorname{cosech}^{2}\left(\frac{m \pi}{\lambda}\right)\right) \\
& =-\frac{\pi^{2}}{\lambda \omega_{1}^{2}} \mu_{2}\left(\frac{1}{\lambda}\right) .
\end{aligned}
$$

Using the relationship between each of these sums and the complementary sum $S_{2}^{*}$, we obtain

so that

$$
S_{2}-S_{2}^{* *}=\frac{4 \pi}{\lambda \omega_{1}^{2}}
$$

$$
F_{1}\left(t ; \omega_{1}, \omega_{2}\right)=F_{1}\left(t ; \omega_{1}^{*}, \omega_{2}^{*}\right)+\frac{4 \pi A}{\lambda \omega_{1}^{2}}
$$


The other parameter $S_{4}$ is independent of the choice of periods and reduces to

$$
\begin{aligned}
S_{4}= & \frac{\pi^{4}}{\omega_{1}^{4}}\left(\frac{1}{45}-\frac{4}{3}\left(\sum_{1}^{\infty} \operatorname{sech}^{2}\left((2 m-1) \pi \frac{\lambda}{2}\right)-\operatorname{cosech}^{2}(m \pi \lambda)\right)\right) \\
& +2\left(\sum_{1}^{\infty} \operatorname{sech}^{4}\left(\frac{(2 m-1) \pi \lambda}{2}\right)+\operatorname{cosech}^{4}(m \pi \lambda)\right)=\frac{\pi^{4}}{\omega_{1}^{4}} \sigma_{4}(\lambda) \\
= & \frac{\pi^{4}}{\lambda^{4} \omega_{1}^{4}} \sigma_{4}\left(\frac{1}{\lambda}\right) .
\end{aligned}
$$

The second case occurs when the period parallelogram is a rectangle with one real period $\omega_{1}$ and one purely imaginary period $\omega_{2}=i \lambda \omega_{1}$, say. Again we can take $\alpha_{1}=-\frac{1}{2} \omega_{1}$ or $\alpha_{2}=\frac{1}{2} \omega_{2}$, although in this case we obtain different functions, since $\alpha_{1}$ and $\alpha_{2}$ do not differ by a period.

With $\alpha=-\frac{1}{2} \omega_{1}$ we have as before that

$$
\Phi\left(t, \omega_{1}\right)=\frac{\pi^{2} A}{\omega_{1}^{2}} \sec ^{2}\left(\frac{\pi t}{\omega_{1}}\right) .
$$

In this case $\Phi\left(t, \omega_{1}\right)$ is also real and is

$$
=\frac{\pi^{2} A}{\lambda^{2} \omega_{1}^{2}} \operatorname{cosech}^{2}\left(\frac{\pi t}{\lambda \omega_{1}}+\frac{\pi}{2 \lambda}\right) .
$$

The double sum is

$$
\begin{aligned}
F_{2}\left(t ; \omega_{1}, \omega_{2}\right) & =\frac{\pi^{2} A}{\omega_{1}^{2}} \sum_{-\infty}^{\infty} \sec ^{2}\left(\frac{\pi t}{\omega_{1}}+m \pi \lambda i\right) \\
& =\frac{\pi^{2} A}{\omega_{1}^{2}}\left(\sec ^{2} \frac{\pi t}{\omega_{1}}+4 \sum_{1}^{\infty} \frac{1+\cos \left(2 \pi t / \omega_{1}\right) \cosh (2 m \pi \lambda)}{\left(\cos \left(2 \pi t / \omega_{1}\right)+\cosh (2 m \pi \lambda)\right)^{2}}\right) .
\end{aligned}
$$

The complementary expansion is

$$
\begin{aligned}
F_{2}\left(t ; \omega_{2}, \omega_{1}\right) & =\frac{\pi^{2} A}{\lambda^{2} \omega_{1}^{2}} \sum_{-\infty}^{\infty} \operatorname{cosech}^{2}\left(\frac{\pi t}{\lambda \omega_{1}}+\left(m+\frac{1}{2}\right) \frac{\pi}{\lambda}\right) \\
& =F_{2}\left(t ; \omega_{1}, \omega_{2}\right)-\frac{2 \pi A}{\lambda \omega_{1}^{2}} .
\end{aligned}
$$

With $\alpha=\frac{1}{2} \omega_{2}$, the function

$$
\Phi\left(t, \omega_{1}\right)=\frac{\pi^{2} A}{\omega_{1}^{2}} \operatorname{cosec}^{2}\left(\frac{\pi t}{\omega_{1}}-i \frac{\lambda \pi}{2}\right)
$$

is not real, but

$$
\Phi\left(t, \omega_{2}\right)=-\frac{\pi^{2} A}{\lambda^{2} \omega_{1}} \operatorname{sech}^{2}\left(\frac{\pi t}{\lambda \omega_{1}}\right)
$$


and we have

and

$$
\begin{aligned}
F_{3}\left(t ; \omega_{1}, \omega_{2}\right) & =\frac{\pi^{2} A}{\omega_{1}^{2}} \sum_{-\infty}^{\infty} \operatorname{cosec}^{2}\left(\frac{\pi t}{\omega_{1}}+\left(m-\frac{1}{2}\right) \lambda \pi i\right) \\
& =\frac{4 \pi^{2} A}{\omega_{1}^{2}} \sum_{1}^{\infty} \frac{1-\cos \left(2 \pi t / \omega_{1}\right) \cosh (2 m-1) \pi \lambda}{\left(\cosh (2 m-1) \pi \lambda-\cos \left(2 \pi t / \omega_{1}\right)\right)^{2}}
\end{aligned}
$$

$$
\begin{aligned}
F_{3}\left(t ; \omega_{2}, \omega_{1}\right) & =-\frac{\pi^{2} A}{\lambda^{2} \omega_{1}^{2}} \sum_{-\infty}^{\infty} \operatorname{sech}^{2}\left(\frac{\pi t}{\lambda \omega_{1}}+\frac{m \pi}{\lambda}\right) \\
& =F_{3}\left(t ; \omega_{1}, \omega_{2}\right)-\frac{2 \pi A}{\lambda \omega_{1}^{2}} .
\end{aligned}
$$

For both these expansions,

$$
\begin{aligned}
S_{4} & =\frac{\pi^{4}}{\omega_{1}^{4}}\left(\frac{1}{45}+\frac{4}{3} \sum_{1}^{\infty} \operatorname{cosech}^{2}(m \pi \lambda)+2 \sum_{1}^{\infty} \operatorname{cosech}^{4}(m \pi \lambda)\right) \\
& =\frac{\pi^{4}}{\lambda^{4} \omega_{1}^{4}}\left(\frac{1}{45}+\frac{4}{3} \sum_{1}^{\infty} \operatorname{cosech}^{2}\left(\frac{m \pi}{\lambda}\right)+2 \sum_{1}^{\infty} \operatorname{cosech}^{2}\left(\frac{m \pi}{\lambda}\right)\right) .
\end{aligned}
$$

These three types of solution are connected by $F_{1}=F_{2}+F_{3}$.

\section{Aperiodic solutions}

All the double sums represent real periodic functions, while the real function $\left(\pi^{2} A / \omega_{1}^{2}\right) \sec ^{2}\left(\pi t / \omega_{1}\right)$ is also periodic. The exceptional cases are the functions $\operatorname{sech}^{2}$ and $\operatorname{cosech}^{2}$, which represent simple sums, and the basic function $f(t)=$ $(t-\alpha)^{-2}$.

These functions correspond to the separatrices in the phase plane trajectories of the system $d y / d t=\mathrm{v}$, where

$$
\frac{d \mathbf{v}}{d t}=a_{2} y^{2}+a_{1} y+a_{0}
$$

When the equation $a_{2} y^{2}+a_{1} y+a_{0}=0$ has no real solutions, there are no singular points in the phase plane, and, as will be shown later, all the solutions of the equation $y^{\prime \prime}=a_{2} y^{2}+a_{1} y+a_{0}$ can be expressed in terms of $F_{1}\left(t ; \omega_{1}, \omega_{2}\right)$. The "periodicity" of these solutions corresponds to the finite time taken for the trajectories to be traversed.

When the equation $a_{2} y^{2}+a_{1} y+a_{0}=0$ has repeated roots, so that $y^{\prime \prime}=$ $a_{2}(y-c)^{2}$, we have, as well as the trajectories of the type $F_{1}$, the degenerate trajectory $\mathbf{v}^{2}=\frac{2}{3} a_{2}(y-c)^{3}$ corresponding to $y=c+6(t-\alpha)^{-2} / a_{2}$, which represents a limiting form of $F_{1}$ as $\left(\omega_{1}, \omega_{2}\right) \rightarrow \infty$. 


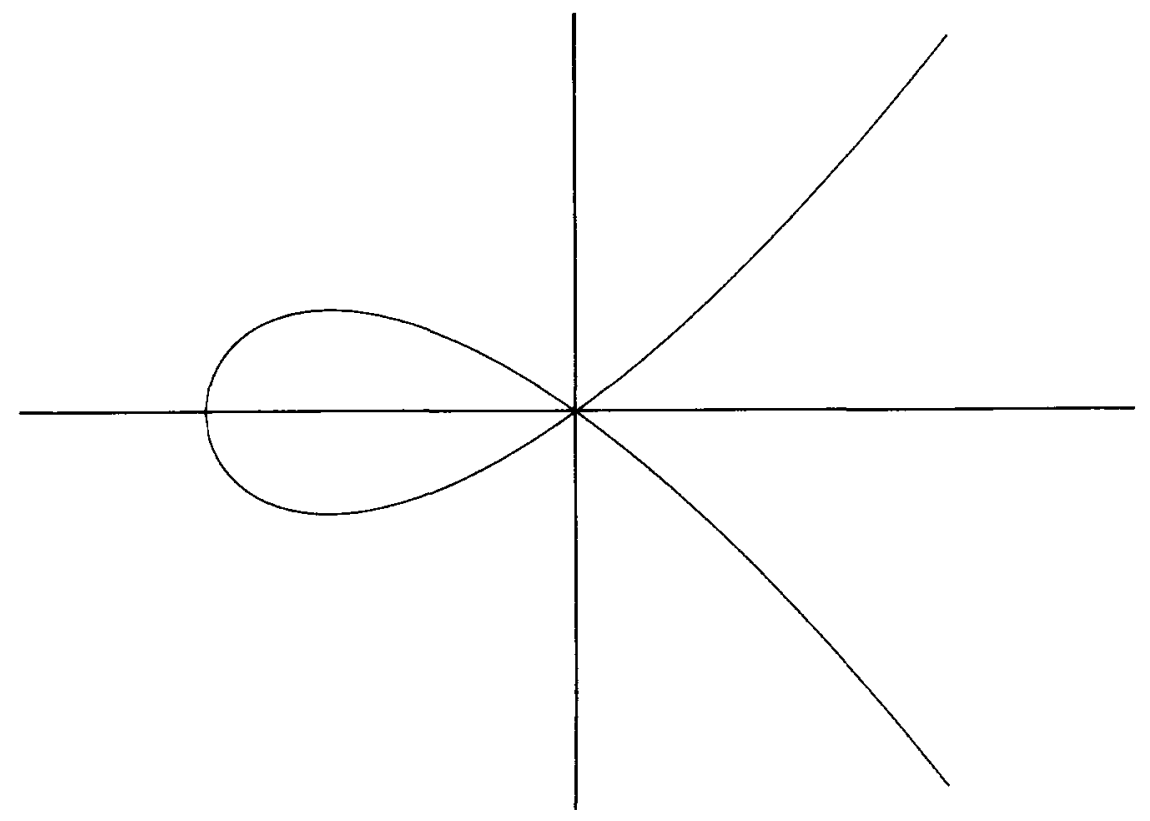

FIGURE 1

Finally, when the equation has two distinct real roots, we obtain separatrices in the form shown in Figure 1.

The closed loop corresponds to a solution of the form $y=-\operatorname{sech}^{2}(\alpha t)$, and the semi-infinite branches correspond to a solution of the form $y=\operatorname{cosech}^{2}(\alpha t)$. The only solutions which are finite and periodic for all $t$ have trajectories lying within the closed loop. They represent solutions in the form $F_{3}\left(t ; \omega_{1}, \omega_{2}\right)$, of which the expansion considered by Whitham is a special case.

$$
\text { Solutions of } y^{\prime \prime}=a_{2} y^{2}+a_{1} y+a_{0}
$$

In order to express the solutions of this equation in terms of the expansions which we have generated, we first scale $y$ by $y=A y$, where $A=6 / a_{2}^{2}$. This gives $y^{\prime \prime}=6 y^{2}+b_{1} Y+b_{0}$, where $b_{1}=a_{1}, b_{0}=a_{0} a_{2} / 6$. If we now put $Y=F+c=$ $p(t-\alpha)+\left(S_{2}+c\right)=p(t-\alpha)+c_{1}$, then $Y^{\prime \prime}=6\left(Y-c_{1}\right)^{2}-30 S_{4}$, but $Y^{\prime \prime}=6\left(Y+b_{1} / 12\right)^{2}+b_{0}-b_{1}^{2} / 24$, so that $S_{4}=\left(b_{1}^{2}-24 b_{0}\right) / 720=$ $\left(a_{1}^{2}-4 a_{0} a_{2}\right) / 720$. The equation $a_{2} y^{2}+a_{1} y+a_{0}=0$ has no real solutions if 
$a_{1}^{2}-4 a_{0} a_{2}<0$ and has repeated real roots if $a_{1}^{2}-4 a_{0} a_{2}=0$. These conditions correspond to the conditions $S_{4}<0$ and $S_{4}=0$, respectively.

However, the form for $S_{4}$, namely

$$
S_{4}=\frac{\pi^{4}}{\omega_{1}^{4}}\left(\frac{1}{45}+\frac{4}{3} \sum_{1}^{\infty} \operatorname{cosech}^{2}(m \pi \lambda)+2 \sum_{1}^{\infty} \operatorname{cosech}^{4}(m \pi \lambda)\right),
$$

which corresponds to functions of the type $F_{2}, F_{3}$, is always positive (although $S_{4} \rightarrow 0$ as $\omega_{1} \rightarrow \infty, \lambda$ finite), so that, when $S_{4}<0$, we only have solutions of the type $F_{1}$ for which $S_{4}<0$ when $1 / \sqrt{3}<\lambda<\sqrt{3}$.

When $S_{4}=0$, we have, apart from the separatrix, solutions of the form $F_{1}$ with $\lambda=1 / \sqrt{3}$ and $\lambda=\sqrt{3}$, those with the smaller value of $\lambda$ lying to the left of the separatrix and those with the larger value to the right. For these values of $\lambda$, the poles of $F_{1}$ are arranged as equilateral triangles.

Finally, when $S_{4}>0$, we obtain all three types of solution. The regions in which the solutions occur are separated by the curves corresponding to $y=\operatorname{sech}^{2}$, $y=\operatorname{cosech}^{2}$ and $y=\sec ^{2}$ (see Figure 2), which represent the limiting forms of the families as $\lambda \rightarrow \infty$ or $\omega \rightarrow \infty, \lambda \omega=$ constant.

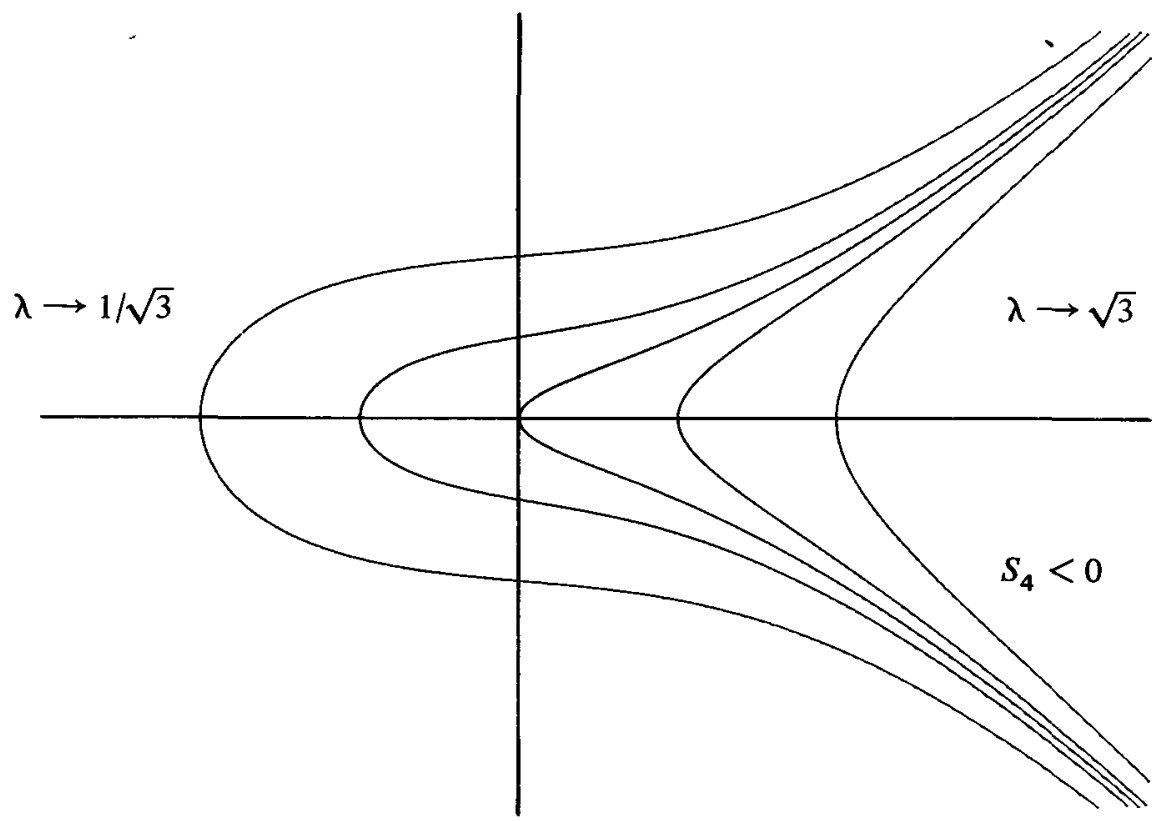

FIGURE 2a 


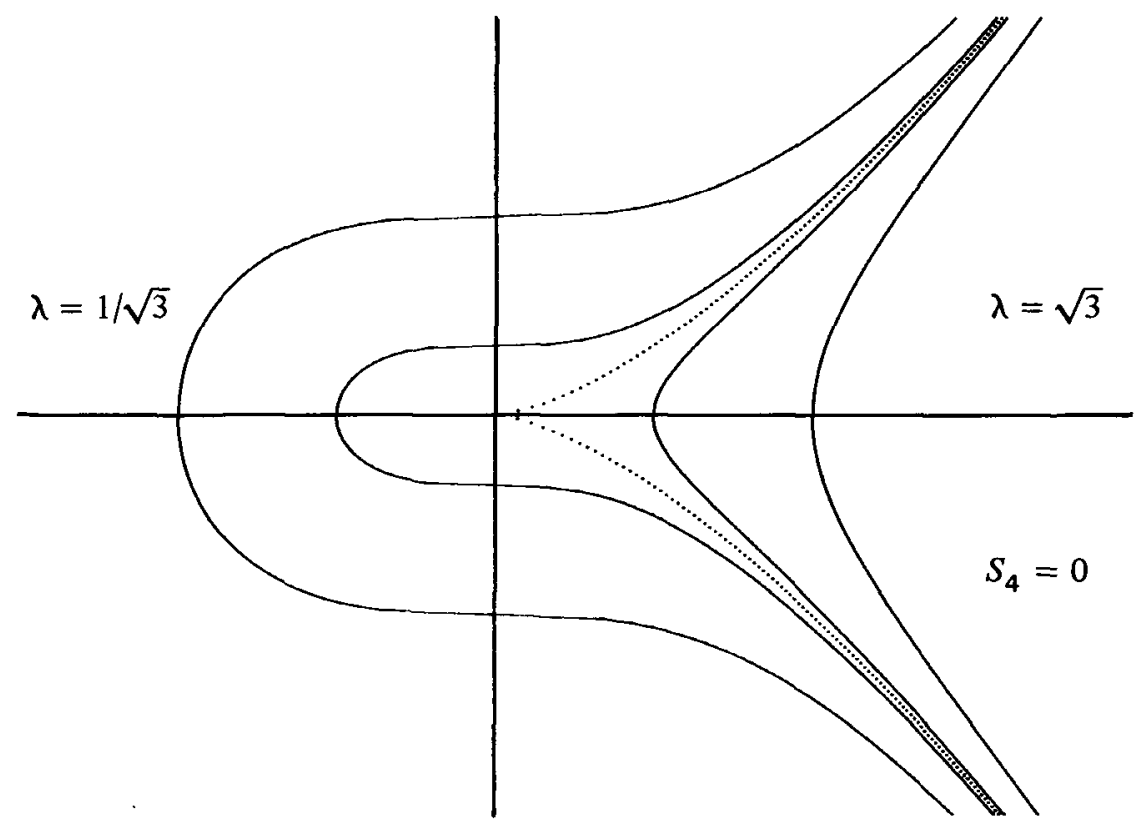

FIguRE 2b

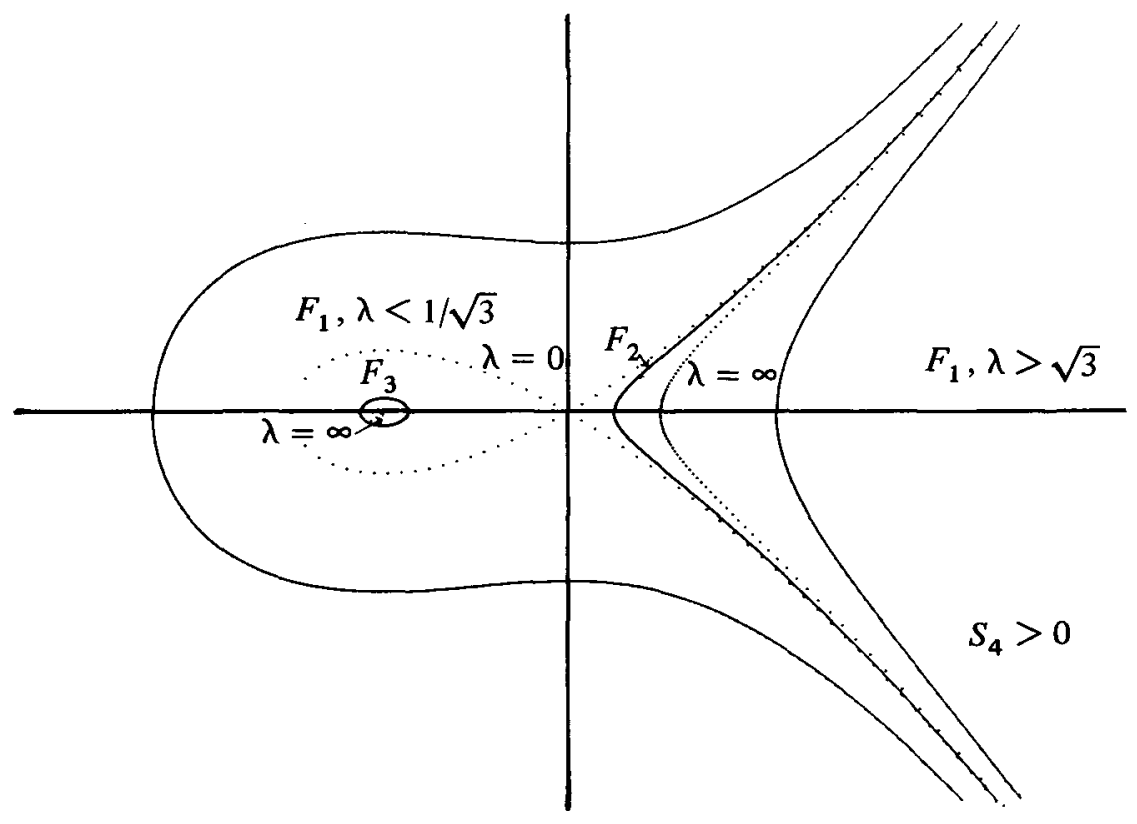

FIGURE 2c 


\section{Functions with simple poles}

The functions $g, \psi, G$, which we have considered, satisfy equations of the form $y^{\prime \prime}=a_{3} y^{3}+a_{2} y^{2}+a_{1} y+a_{0}$ with $a_{3}=2 / A^{2}$. Hence, if we are looking for real solutions as functions of a real variable, there are two distinct cases depending on whether $a_{3}>0$ or $a_{3}<0$.

These two cases are distinguished by the behaviour of their trajectories in the phase plane. When $a_{3}<0$, all the trajectories in the phase plane are closed, and almost all the solutions are finitely periodic. On the other hand, when $a_{3}>0$, there are finitely periodic solutions only when the polynomial has three distinct real roots, and then only a few of the solutions have this form.

\section{The case $a_{3}<0$}

In order to reduce the number of parameters involved, we will assume that $A=-i$, so that all the functions developed satisfy an equation with $a_{3}=-2$.

For our basis function we take $g(t)=2 \beta\left[(t-\alpha)^{2}+\beta^{2}\right]^{-1}$ and develop from it three families of solutions similar in structure to those developed for the quadratic case. This involves (i) taking $\omega_{1}$ real, $\omega_{2}=\frac{1}{2} \omega_{1}(1+i \lambda), \alpha=\frac{1}{2} \omega_{1}$ (or equally $\omega_{1}^{*}=i \lambda \omega_{1}, \omega_{2}^{*}=\omega_{2}, \alpha=\frac{1}{2} \omega_{1}^{*}$ ); (ii) taking $\omega_{1}$ real, $\omega_{2}=i \lambda \omega_{1}, \alpha=\frac{1}{2} \omega_{1}$; and (iii) taking $\omega_{1}$ real, $\omega_{2}=i \lambda \omega_{1}, \alpha=\frac{1}{2} \omega_{2}$. From (i) we obtain

$$
\begin{aligned}
& \psi_{1}\left(t ; \omega_{1}, \beta\right)=\frac{2 \pi}{\omega_{1}} \frac{\sinh \left(2 \pi \beta / \omega_{1}\right)}{\cosh \left(2 \pi \beta / \omega_{1}\right)+\cos \left(2 \pi t / \omega_{1}\right)} \\
& \psi_{2}\left(t ; \omega_{1}^{*} ; \beta\right)=-\frac{2 \pi}{\lambda \omega_{1}} \frac{\sin \left(2 \pi \beta / \lambda \omega_{1}\right)}{\cos \left(2 \pi \beta / \lambda \omega_{1}\right)+\cosh \left(2 \pi t / \lambda \omega_{1}\right)}
\end{aligned}
$$

and the double sums

$$
\begin{aligned}
G_{1}\left(t ; \omega_{1}, \omega_{2}, \beta\right) & =\frac{2 \pi}{\omega_{1}} \sum_{-\infty}^{\infty} \frac{\sinh \left(2 \pi \beta / \omega_{1}\right)}{\cosh \left(2 \pi \beta / \omega_{1}\right)+(-1)^{m} \cos \left(2 \pi t / \omega_{1}+m \pi \lambda i\right)} \\
G_{1}\left(t ; \omega_{1}^{*}, \omega_{1}^{*} ; \beta\right) & =-\frac{2 \pi}{\lambda \omega_{1}} \sum_{-\infty}^{\infty} \frac{\sin \left(2 \pi \beta / \lambda \omega_{1}\right)}{\cos \left(2 \pi \beta / \lambda \omega_{1}\right)+(-1)^{m} \cosh \left(2 \pi t / \lambda \omega_{1}+m \pi / \lambda\right)} \\
& =G_{1}\left(t ; \omega_{1}, \omega_{2} ; \beta\right)-\frac{4 \pi \beta}{\lambda \omega_{1}^{2}} .
\end{aligned}
$$

Setting $\omega_{1}=\beta w_{1}, \omega_{2}=\beta w_{2}, t=\beta \tau$, we see that

$$
G_{1}\left(t ; \omega_{1}, \omega_{2} ; \beta\right)=\frac{1}{\beta} G\left(\tau ; w_{1}, w_{2} ; 1\right),
$$


so that $\beta$ represents a scale factor with respect to the family of solutions. While $\beta$ can theoretically range from $-\infty$ to $\infty$, there is no loss of generality in assuming that $0<\beta \leqslant \lambda \omega / 2$, since, except, for $\psi_{1}, \beta+m \lambda \omega$ gives the same function values as $\beta$ to within a constant, and replacing $\beta$ by $-\beta$ merely changes the sign of the function.

When $\beta=\lambda \omega_{1} / 2, G$ reduces to a constant, and we have the functional identity

$$
\sum_{-\infty}^{\infty} \frac{\sinh (\pi \lambda)}{\cosh (\pi \lambda)+(-1)^{m} \cos \left(2 \pi t / \omega_{1}+m \pi \lambda i\right)}=1 .
$$

$\psi_{1}$ represents the limit of $G_{1}$ as $\lambda \rightarrow \infty$. However, as $\lambda \rightarrow 0$, we obtain a two piece limiting function. If we take the limit with $t$ unchanged, we obtain $G_{1} \rightarrow \psi_{2}$ as $\lambda \rightarrow 0, \lambda \omega_{1}$ finite. However, if we first shift $t$ by $\tau=t-\omega_{1} / 2$, we obtain the limit

$$
G_{1} \rightarrow-\frac{2 \pi}{\lambda \omega_{1}} \frac{\sin \left(2 \pi \beta / \lambda \omega_{1}\right)}{\cos \left(2 \pi \beta / \lambda \omega_{1}\right)-\cosh \left(2 \pi \tau / \omega_{1}\right)},
$$

which represents a closed loop in the right half plane.

Similarly, from (ii) we obtain

$$
G_{2}\left(t ; \omega_{1}, \omega_{2} ; \beta\right)=\frac{2 \pi}{\omega_{1}} \sum_{-\infty}^{\infty} \frac{\sinh \left(2 \pi \beta / \omega_{1}\right)}{\cosh \left(2 \pi \beta / \omega_{1}\right)+\cos \left(2 \pi t / \omega_{1}+2 m \pi \lambda i\right)}
$$

and

$$
\begin{aligned}
G_{2}\left(t ; \omega_{2}, \omega_{1} ; \beta\right) \\
\quad=-\frac{2 \pi}{\lambda \omega_{1}} \sum_{-\infty}^{\infty} \frac{\sin \left(2 \pi \beta / \lambda \omega_{1}\right)}{\cos \left(2 \pi \beta / \lambda \omega_{1}\right)-\cosh \left(2 \pi t / \lambda \omega_{1}+(2 m-1) \pi / \lambda\right)} \\
\quad=G_{2}\left(t ; \omega_{1}, \omega_{2} ; \beta\right)-\frac{2 \pi \beta}{\lambda \omega_{1}^{2}} .
\end{aligned}
$$

In this case $G_{2} \rightarrow \psi_{1}$ as $\lambda \rightarrow \infty$, but in order to obtain the other limit we need first to set $\tau=t-\omega_{1} / 2$ to obtain

$$
\begin{aligned}
G_{2} & \rightarrow-\frac{2 \pi}{\lambda \omega_{1}} \frac{\sin \left(2 \pi \beta / \lambda \omega_{1}\right)}{\cos \left(2 \pi \beta / \lambda \omega_{1}\right)-\cosh \left(2 \pi t / \lambda \omega_{1}\right)} \text { as } \lambda \rightarrow 0, \lambda \omega \text { finite, } \\
& =-\psi_{2}\left(\tau ; \omega_{1}^{*} ;-\beta+\frac{\lambda \omega_{1}}{2}\right),
\end{aligned}
$$

which represents the right hand branch of the $G_{1}$ limit.

For the third case we obtain

$$
\begin{array}{r}
G_{3}\left(t ; \omega_{1}, \omega_{2} ; \beta\right)=\frac{2 \pi}{\omega_{1}} \sum_{-\infty}^{\infty} \frac{\sinh \left(2 \pi \beta / \lambda \omega_{1}\right)}{\cosh \left(2 \pi \beta / \omega_{1}\right)-\cos \left(2 \pi t / \omega_{1}+(2 m-1) \pi \lambda i\right)} \\
=\frac{2 \pi \beta}{\lambda \omega_{1}^{2}}-\frac{2 \pi}{\lambda \omega_{1}} \sum_{-\infty}^{\infty} \frac{\sin \left(2 \pi \beta / \lambda \omega_{1}\right)}{\cos \left(2 \pi \beta / \lambda \omega_{1}\right)+\cosh \left(2 \pi t / \lambda \omega_{1}+2 m \pi / \lambda\right)} .
\end{array}
$$


In this case $G_{3} \rightarrow 0$ as $\lambda \rightarrow \infty$, while $G_{3} \rightarrow \psi_{2}$ as $\lambda \rightarrow 0, \lambda \omega$ finite, which represents the left hand branch of the $G_{1}$ limit. As with the quadratic case these families are connected by $G_{1}=G_{2}+G_{3}$.

Note also that when $\beta / \lambda \omega_{1}=\frac{1}{4}$, we have

$$
G_{2}\left(t ; \omega_{1}, \omega_{2} ; \beta\right)=\frac{2 \pi}{\lambda \omega_{1}} \sum_{-\infty}^{\infty} \operatorname{sech}\left(\frac{2 \pi}{\lambda \omega_{1}}\left(t-\frac{\omega_{1}}{2}\right)+\frac{2 m \pi}{\lambda}\right)
$$

and

$$
G_{2}\left(t ; \omega_{2}, \omega_{1} ; \beta\right)=-\frac{2 \pi}{\lambda \omega_{1}} \sum_{-\infty}^{\infty} \operatorname{sech}\left(\frac{2 \pi}{\lambda \omega_{1}} t+\frac{2 m \pi}{\lambda}\right),
$$

so that the trajectories of one family are the images of the other. This case is also significant for the family $G_{1}$, since when $\beta / \lambda \omega_{1}=\frac{1}{4}$ the trajectories are symmetric about both axes.

$$
\text { Solutions of } y^{\prime \prime}=a_{3} y^{3}+a_{2} y^{2}+a_{1} y+a_{0}
$$

In order to determine the regions in the phase plane to which these families of solutions correspond, we first reduce the equation to a collection of standard forms.

Firstly we scale the variable $y$ by $y=A \eta$, choosing $A$ so that the leading coefficient becomes -2 , and then we shift $\eta$ by $\eta=z-c$ in order to eliminate the second order term in the polynomial. This gives

$$
z^{\prime \prime}=-2 z^{3}+\left(a_{1}-\frac{a_{2}^{2}}{3 a_{1}}\right) z \pm \sqrt{\frac{-a_{3}}{2}}\left(\frac{2 a_{2}^{3}}{27 a_{3}^{2}}-\frac{a_{1} a_{2}}{3 a_{3}}+a_{0}\right),
$$

where the \pm corresponds to the choice of sign when determining $A$.

When $a_{1}=a_{2}^{2} / 3 a_{3}$, the equation has the standard form

$$
z^{\prime \prime}=-2 z^{3}+b_{0}, \quad \text { where } b_{0}= \pm \sqrt{\frac{-a_{3}}{2}}\left(a_{0}-\frac{a_{2}^{3}}{2 z a_{3}^{2}}\right) .
$$

Otherwise we can scale $z$ and $t$ simultaneously by $z=r Y, t=\tau / r$, where $r^{2}=\left|a_{1}-a_{2}^{2} / 3 a_{3}\right|$, to reach the forms

$$
Y^{\prime \prime}=-2 Y^{3} \pm Y+b_{0}
$$

depending on the sign of $a_{1}-a_{2}^{2} / 3 a_{3}$. The sign of $b_{0}$ is not of consequence, since replacing $Y$ by $-Y$ replaces $b_{0}$ by $-b_{0}$. However, the magnitude of $b_{0}$ is relevant 
when the right hand side is $-2 Y^{3}+Y+b_{0}$, since the number of distinct real roots of this polynomial is 3,2 or 1 , depending on whether $b_{0}^{2}$ is $<,=$, or $>2 / 27$.

\section{Limiting forms}

When studying the quadratic case, we saw that the regions corresponding to the different families of solutions were separated by the more basic solutions which represented the limiting forms as one or both of the periods become infinite.

For the cubic case with $a_{3}<0$, these forms are

$$
\begin{aligned}
& g(t)=\frac{2 \beta}{6^{2}+\beta^{2}} \\
& \begin{aligned}
\psi_{1}\left(t ; \omega_{1} ; \beta\right) & =\frac{2 \pi}{\omega_{1}} \frac{\sinh \left(2 \pi \beta / \omega_{1}\right)}{\cosh \left(2 \pi \beta / \omega_{1}\right)+\cos \left(2 \pi t / \omega_{1}\right)} \\
& =\frac{\theta \sinh (\beta \theta)}{\cosh (\beta \theta)+\cos (\theta t)}
\end{aligned}
\end{aligned}
$$

and

$$
\begin{aligned}
\psi_{2}\left(t ; \omega_{1}^{*} ; \beta\right) & =-\frac{2 \pi}{\lambda \omega_{1}} \frac{\sin \left(2 \pi \beta / \lambda \omega_{1}\right)}{\cos \left(2 \pi \beta / \lambda \omega_{1}\right)+\cosh \left(2 \pi t / \lambda \omega_{1}\right)} \\
& =-\frac{\phi \sin (\beta \phi)}{\cos (\beta \phi)+\cosh (\phi t)} .
\end{aligned}
$$

In the first case, if $y=2 \beta /\left(t^{2}+\beta^{2}\right)$, then $y^{\prime \prime}=-2 y^{3}+3 y^{2} / \beta$, so that, upon setting $Y=y-1 / \beta, 2 \beta^{2}=3$, we have $Y^{\prime \prime}=-2 Y^{3}+Y \pm 2 / 3 \sqrt{6}$. Therefore this solution represents the limiting form for the case $b_{0}^{2}=2 / 27$. Similarly, if

$$
Y=\frac{\theta \sinh (\beta \theta)}{\cosh (\beta \theta)+\cos (\theta t)}-\frac{1}{2} \theta \operatorname{coth}(\beta \theta),
$$

then we have

$$
Y^{\prime \prime}=-2 Y^{3}+\theta^{2}\left(\frac{3}{2} \operatorname{coth}^{2}(\beta \theta)-1\right) Y+\frac{1}{2} \theta^{3} \operatorname{coth}(\beta \theta)\left(\operatorname{coth}^{2}(\beta \theta)-1\right) .
$$

Since $\frac{3}{2} \operatorname{coth}^{2}(\beta \theta)-1>\frac{1}{2}$, this equation can only be reduced to the form $Y^{\prime \prime}=-2 Y^{3}+Y+b_{0}$ by taking $\theta^{2}\left(\frac{3}{2} \operatorname{coth}^{2}(\beta \theta)-1\right)=1$, which gives $\operatorname{coth}^{2}(\beta \theta)=\frac{2}{3}\left(1+\theta^{-2}\right)$ for $0 \leqslant \theta \leqslant 2$. Substituting in the constant term, we obtain

$$
b_{0}=\frac{1}{3 \sqrt{6}}\left(1+\theta^{2}\right)^{1 / 2}\left(2-\theta^{2}\right)
$$


so that $b_{0}^{2} \leqslant 2 / 27$ with equality when $\theta=0$, i.e. when $\omega_{1} \rightarrow \infty$.

In the same way, if

$$
Y=-\frac{1}{2} \phi \cot (\beta \phi)-\phi \frac{\sin \beta \phi}{\cos \beta \phi+\cosh (\phi t)},
$$

then we have

$$
Y^{\prime \prime}=-2 Y^{3}+\phi^{2}\left(\frac{3}{2} \cot ^{2}(\beta \phi)+1\right) Y+\frac{1}{2} \phi^{3} \cot ^{2}(\beta \phi)\left(\cot ^{2}(\beta \phi)+1\right),
$$

which reduces to $Y^{\prime \prime}=-2 Y^{3}+Y+b_{0}$ when $\cot ^{2}(\beta \phi)=\frac{2}{3}\left(\phi^{-2}-1\right), 0 \leqslant \phi^{2}$ $\leqslant 1$, and

$$
b_{0}=\frac{1}{3 \sqrt{6}}\left(1-\phi^{2}\right)^{1 / 2}\left(2+\phi^{2}\right) .
$$

Again we have $b_{0}^{2} \leqslant 2 / 27$, with equality when $\phi=0$, i.e. when $\lambda \omega_{1} \rightarrow \infty$.

Hence the three families of solutions co-exist only for the equation $Y^{\prime \prime}=$ $-2 Y^{3}+Y+b_{0}$ when $b_{0}^{2}<2 / 27$.

Given $b_{0}$ with $b_{0}^{2}<2 / 27$, we can solve these equations to obtain the appropriate values of $\theta$ and $\phi$ for the separating curves. In the first case there is essentially only one solution, since replacing $\theta$ by $-\theta$ does not alter the form of $y$, but in the second case there are two essentially different values of $\beta$, say $\beta_{0}$ and $\beta_{1}$, which are connected by $\beta_{1} \phi=\beta_{0} \phi+\pi$. These values give the two branches of the separatrix which pass through the saddle point in the phase plane, and the branches correspond to the distinct limiting behaviour of the families $G_{2}$ and $G_{3}$ relative to that of $G_{1}$.

Graphs of these separating curves are shown in Figure 3 for the case $b_{0}=$ $1 / 3 \sqrt{3}$, together with the regions to which the families correspond. When $b_{0}=0$, we have $\phi^{2}=1$ and $\cot (\beta \phi)=0$, which corresponds to the case $\beta / \lambda \omega_{1}=\frac{1}{4}$. This is precisely the case, already noted, for which the families $G_{2}$ and $G_{3}$ are symmetric about the saddle point, and it corresponds to the transition from the regions, illustrated above, for $b_{0}>0$ to the reflected picture which occurs for $b_{0}<0$. In this case the outer separators reduce to $y= \pm \operatorname{sech} t$, while the inner separator(s) reduce to $y= \pm 1 / \sqrt{2}$, the singular points of the equation.

When $b_{0}^{2}=2 / 27$, all the separating curves reduce to the single curve corresponding to $Y=2 \beta /\left(t^{2}+\beta^{2}\right)-1 / \beta$, and the regions containing the families $G_{2}$ and $G_{3}$ vanish.

Finally, for $b_{0}^{2}>2 / 27$, there are no separating curves, and all the solutions belong to the $G_{1}$ family. This behaviour mimics that of the quadratic equation, the cases $b_{0}^{2}<,=$, and $>2 / 27$ giving phase diagrams and familiar behaviour similar to that for the cases $S_{4}>$, $=$, and $<0$. 


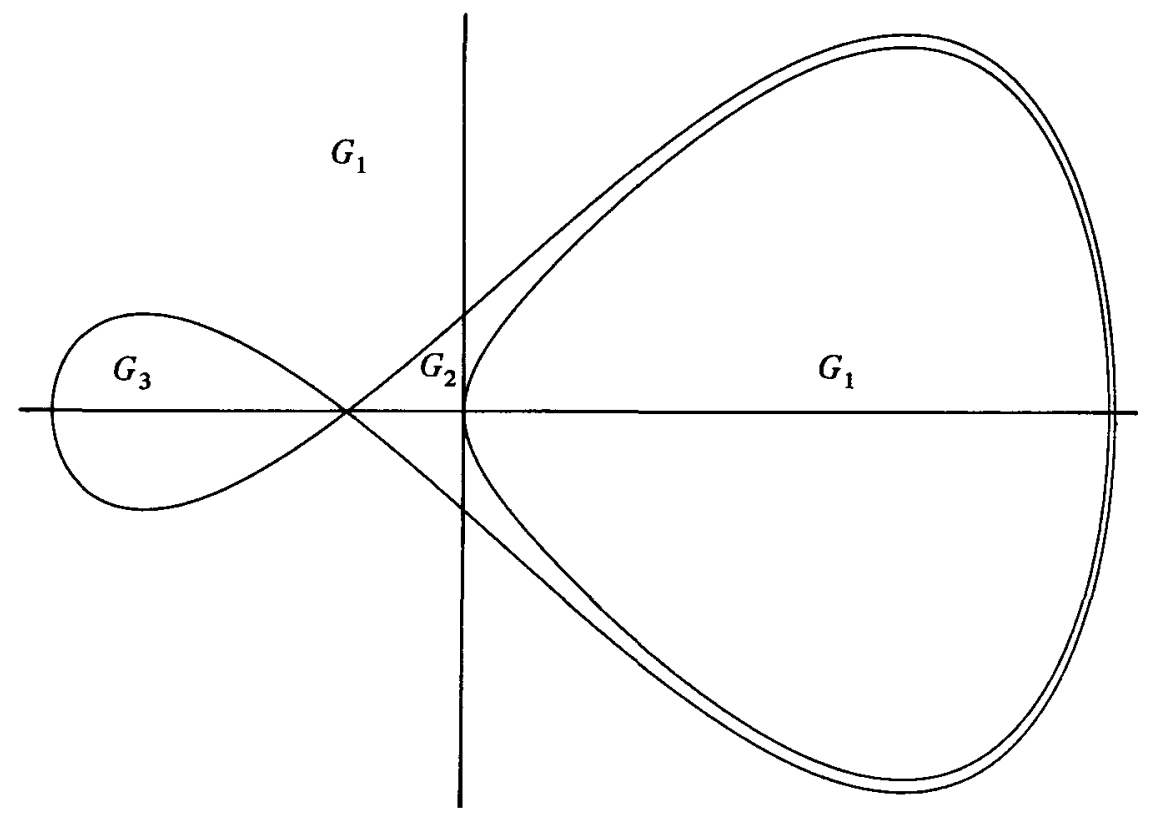

FIGURE 3

The equations $Z^{\prime \prime}=-2 Z^{3}+b_{0}$ and $Y^{\prime \prime}=-2 Y^{3}-Y+b_{0}$ only ever have one real singular point in the phase plane, and their solutions all belong to the family $G_{1}$ for suitable values of the parameter. In particular, the solutions of $Z^{\prime \prime}=-2 Z^{3}$ can be represented in terms of the lemniscate sine sl as $Z=$ $a \operatorname{sl}\left(a\left(t-t_{0}\right)\right)$. They correspond to the function $G_{1}$ with $\lambda=1$ and $\beta=\omega_{1} / 4$.

In summary, for the case $a_{3}<0$, there are two basic soliton solutions

$$
\psi_{1}=\frac{-\phi \sin \beta \phi}{\cos (\beta \phi)+\cosh (\phi t)}
$$

and

$$
\psi_{2}=\frac{\phi \sin (\beta \phi)}{\cosh (\phi t)-\cos (\beta \phi)},
$$

both of which satisfy the same equation. Infinite trains of the $\psi_{1}$ solitons give periodic solutions of the type $G_{3}$, while trains of the $\psi_{2}$ solitons give solutions of the type $G_{2}$. However, as we have seen, solutions of this type are comparatively rare, and most solutions are formed as alternating trains of both types of solitons. Graphs of typical examples of both solitons and the $G_{1}$ solutions obtained by superposition are given in Figure 4. 


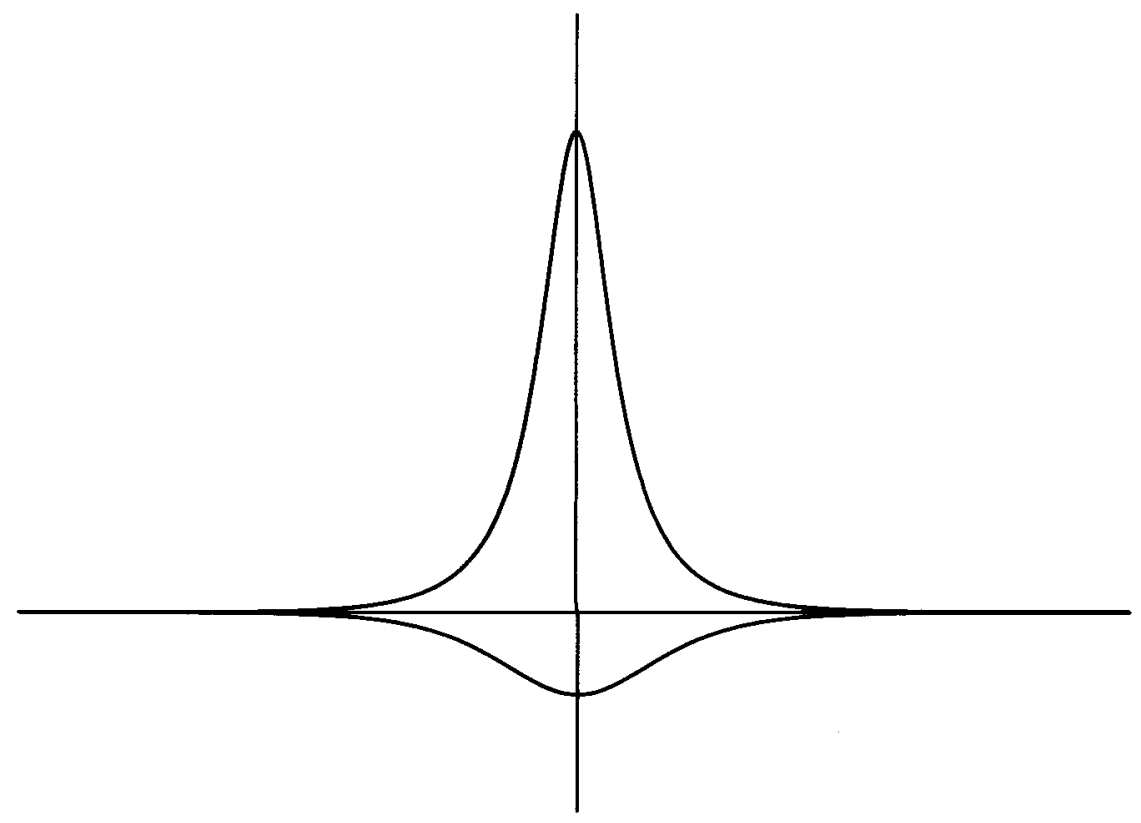

FIGURE 4a

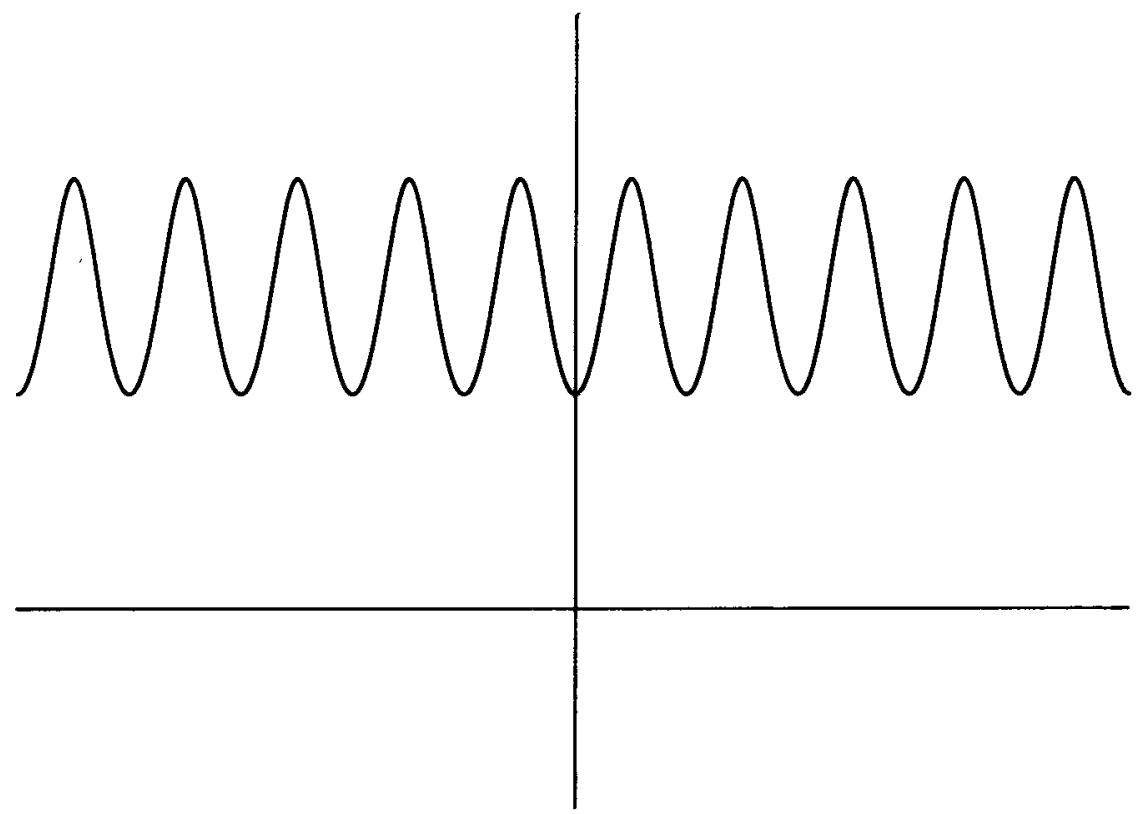

Figure 4b 


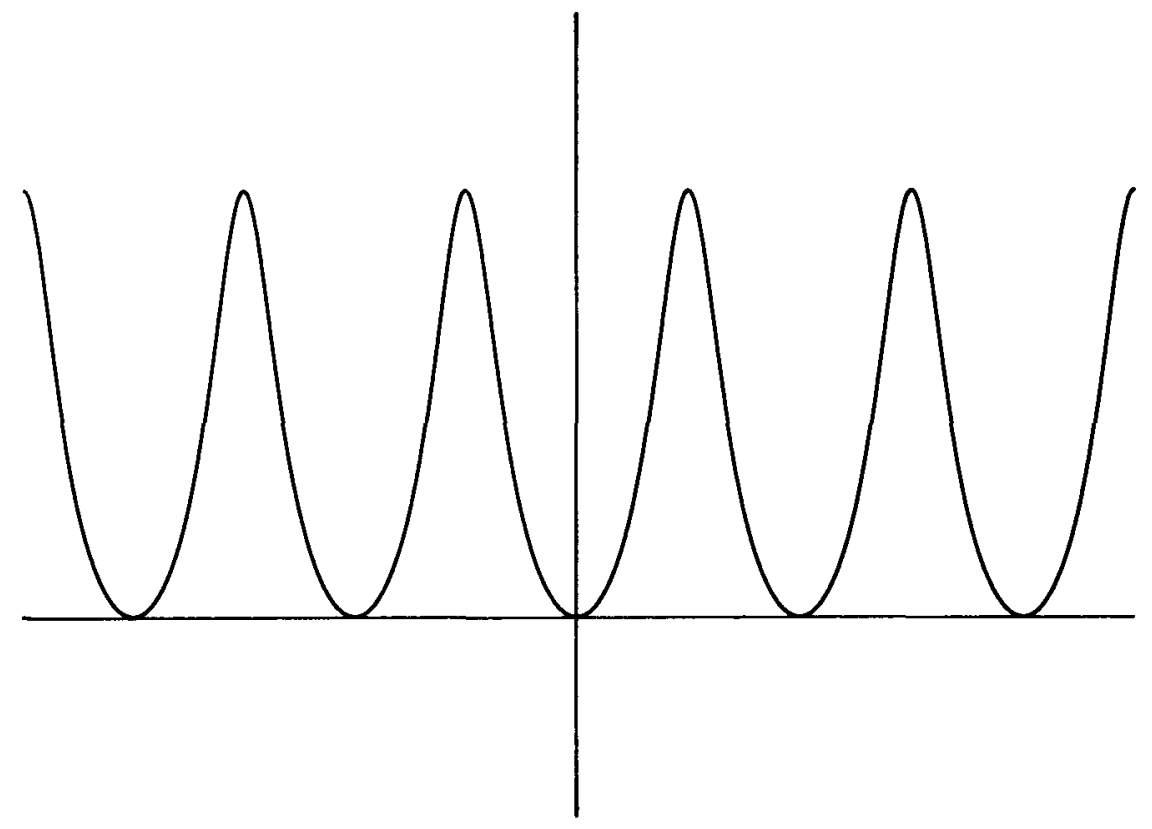

FIGURE 4c

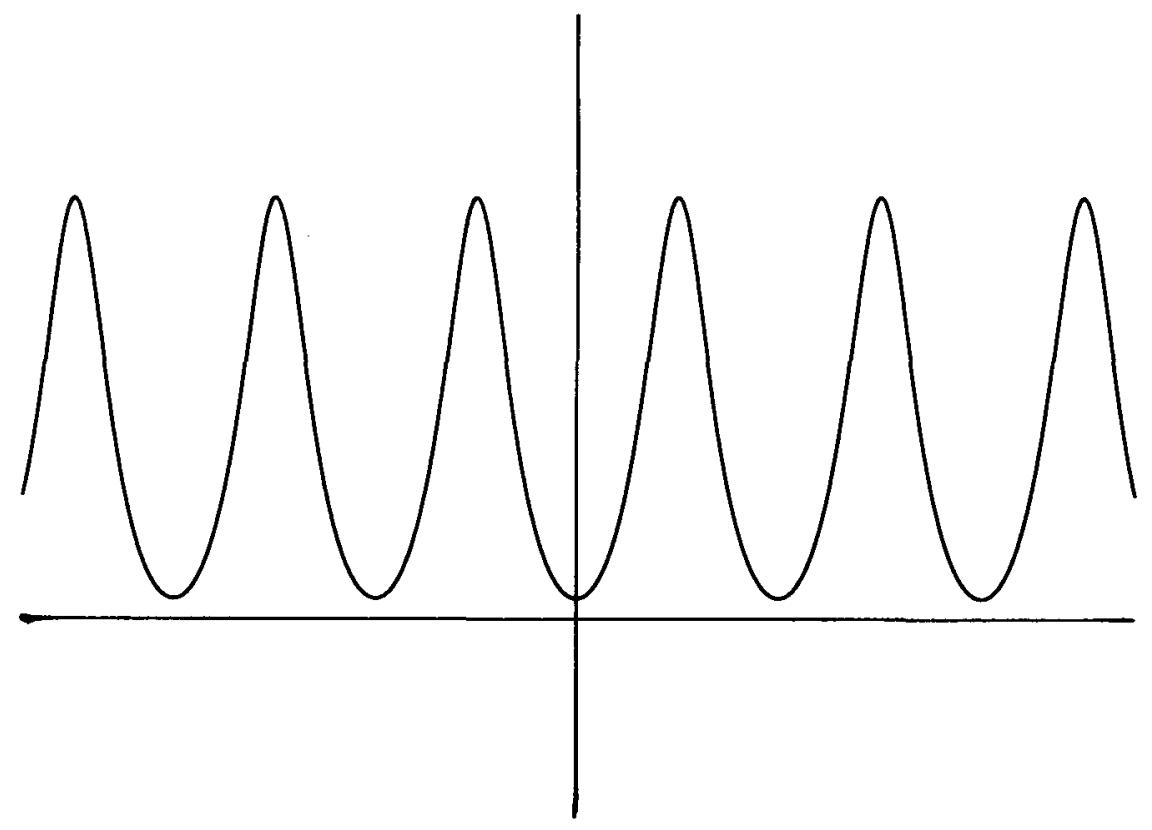

Figure 4d 


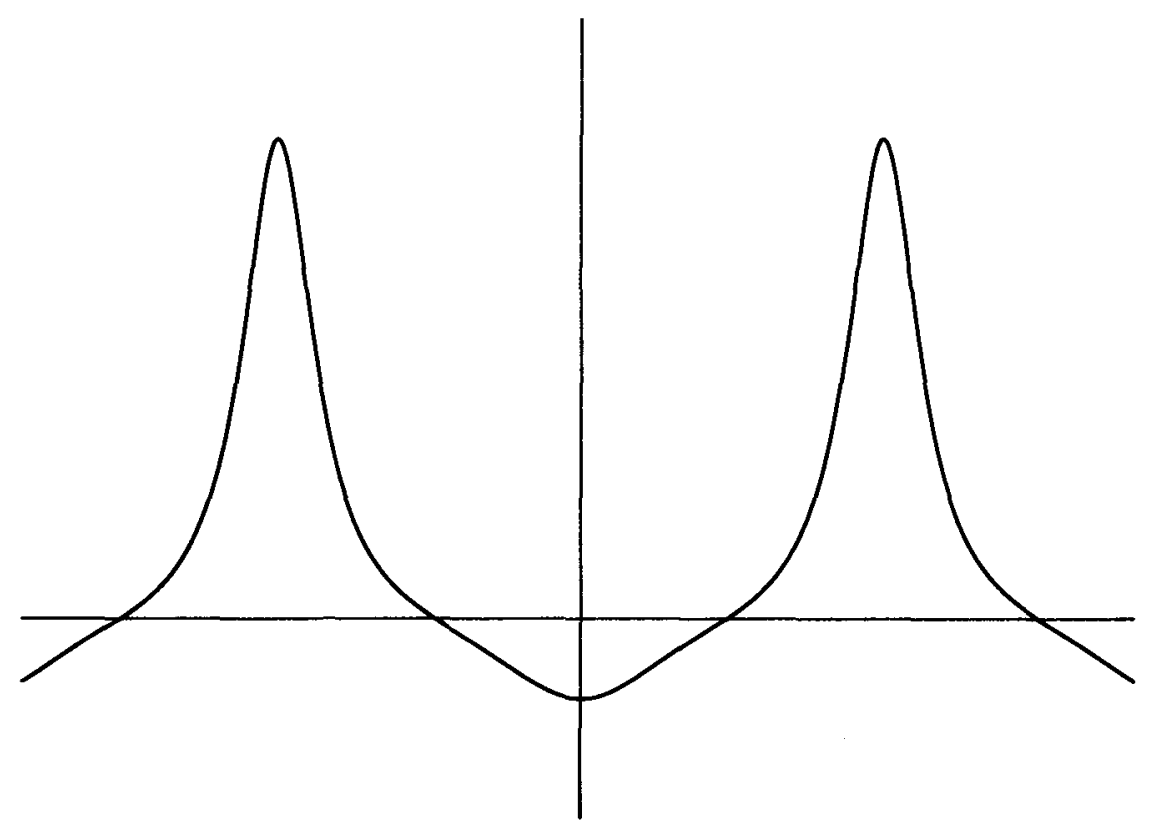

Figure 4e

The case $a_{3}>0$

As with the case $a_{3}<0$, we can develop three families of solutions starting in this case with the basic function $g(t)=2 \beta /\left[(t-\alpha)^{2}-\beta^{2}\right]$. Setting $\theta=2 \pi / \omega_{1}$ and $\phi=2 \pi / \lambda \omega_{1}$, we define these families as follows:

$$
\begin{aligned}
G_{1}\left(t ; \omega_{1} \omega_{2} ; \beta\right) & =\theta \sum_{-\infty}^{\infty} \frac{\sin (\beta \theta)}{\cos (\beta \theta)+(-1)^{m} \cos (\theta t+m \pi \lambda i)} \\
& =\frac{4 \pi \beta}{\lambda \omega_{1}^{2}}-\phi \sum_{-\infty}^{\infty} \frac{\sinh (\beta \phi)}{\cosh (\beta \phi)+(-1)^{m} \cosh (\phi t+m \pi / \lambda)} \\
G_{2}\left(t ; \omega_{1}, \omega_{2} ; \beta\right) & =\theta \sum_{-\infty}^{\infty} \frac{\sin (\beta \theta)}{\cos (\beta \theta)+\cos (\theta t+2 m \pi \lambda i)} \\
& =\frac{2 \pi \beta}{\lambda \omega_{1}^{2}}-\phi \sum_{-\infty}^{\infty} \frac{\sinh (\beta \phi)}{\cosh (\beta \phi)-\cosh (\phi t+(2 m-1) \pi / \lambda)}
\end{aligned}
$$


and

$$
\begin{aligned}
G_{3}\left(t ; \omega_{1}, \omega_{2} ; \beta\right) & =\theta \sum_{-\infty}^{\infty} \frac{\sin \beta \theta}{\cos (\beta \theta)-\cos (\theta t(2 m-1) \pi \lambda i)} \\
& =\frac{2 \pi \beta}{\lambda \omega_{1}^{2}}-\phi \sum_{-\infty}^{\infty} \frac{\sinh (\beta \phi)}{\cosh (\beta \phi)+\cosh (\phi t+2 m \pi / \lambda)}
\end{aligned}
$$

Once again we have $G_{1}=G_{2}+G_{3}$, and for this case only $G_{3}$ gives a finite periodic function.

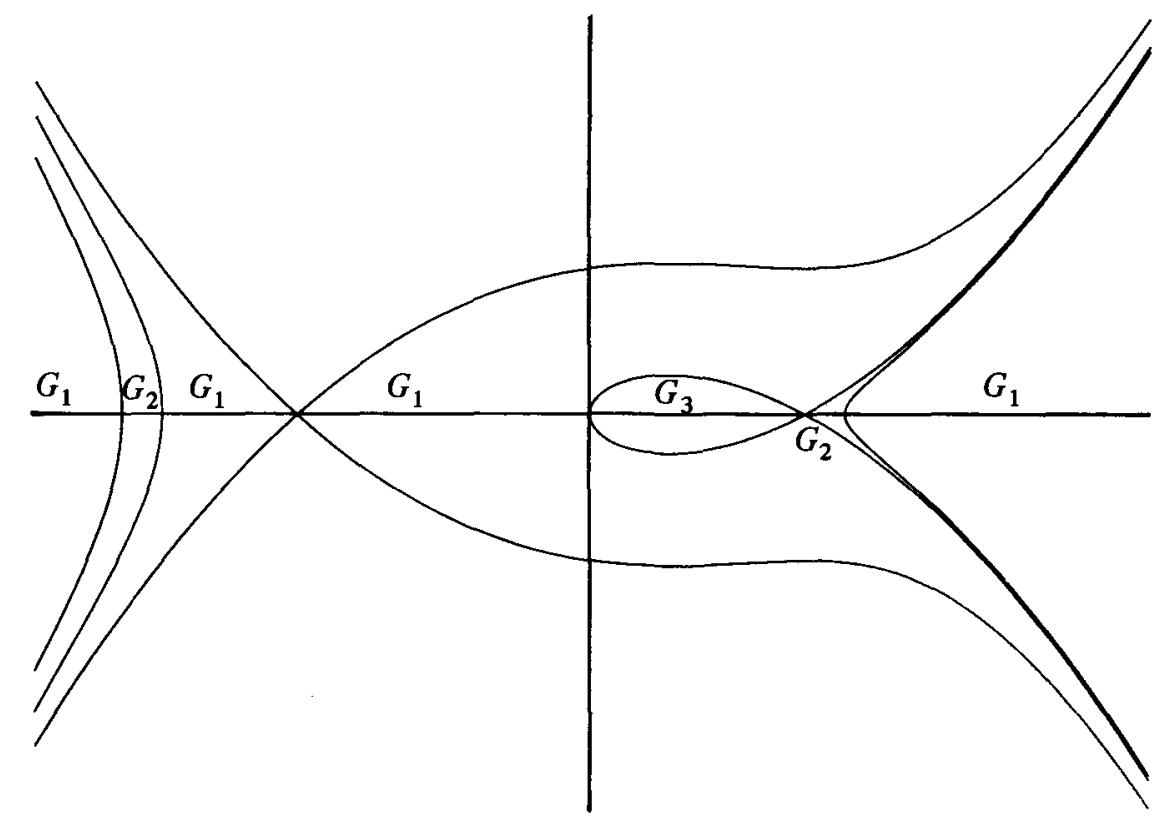

FIGURE 5

The phase plane trajectories of these families of solutions have similar behaviour to those for the earlier case. The families $G_{2}$ and $G_{3}$ occur only when the cubic equation has three distinct real roots (see Figure 5), while members of the family $G_{1}$ occur as solutions for all forms of the right hand side. However, all these solutions are even functions of $t$, so that the derivatives vanish when $t=0$ (and when $t=\frac{1}{2} \omega_{1}$ ); moreover, their trajectories are symmetric about the $y$-axis.

This behaviour is associated with the alternation of the signs of the residues do not vanish, it is necessary to take special forms of $g(t)=(t-a)^{-1}-(t-b)^{-1}$ 
together with appropriately chosen periods so that the poles on the real axis all have the same residue. This can be achieved in two ways.

Firstly, we let $g(t)=t^{-1}-(t+2 i \beta)^{-1}$, and choose $\omega_{1}$ real, $\omega_{2}=4 i \beta$. With this choice, we have $\psi\left(t ; \omega_{2}\right)=\pi / 2 \beta \operatorname{cosech}(\pi t / 2 \beta)$, and the double sum is

$$
G\left(t ; \omega_{2}, \omega_{1} ; \beta\right)=(\pi / 2 \beta) \sum_{-\infty}^{\infty} \operatorname{cosech}\left(\pi t / 2 \beta+m \pi \omega_{1} / 2 \beta\right) .
$$

Alternatively, $\psi\left(t ; \omega_{1}\right)=\left(\pi / \omega_{1}\right)\left(\cot \left(\pi t(t+2 \beta i) / \omega_{1}\right)\right)$, and

$$
\begin{aligned}
G\left(t ; \omega_{1} ; \omega_{2} ; \beta\right) & =\frac{\pi}{\omega_{1}}\left(\cot \left(\frac{\pi t}{\omega_{1}}\right)+\sum_{1}^{\infty} \frac{(-1)^{m} \sin \left(2 \pi t / \omega_{1}\right)}{\sin ^{2}\left(\pi t / \omega_{1}\right)+\sinh ^{2}\left(2 \pi m \beta / \omega_{1}\right)}+i\right) \\
& =G\left(t ; \omega_{2}, \omega_{1} ; \beta\right)+\frac{i \pi}{\omega_{1}}
\end{aligned}
$$

Notice that

$$
\frac{d G}{d t}=-\left(\frac{\pi}{2 \beta}\right)^{2} \sum_{-\infty}^{\infty} \frac{\cosh \left(\pi t / 2 \beta-m \pi \omega_{1} / 2 \beta\right)}{\sinh ^{2}\left(\pi t / 2 \beta+m \pi \omega_{1} / 2 \beta\right)}<0
$$

as required. These solutions are odd functions of $t$, so that they satisfy equations of the form

$$
y^{\prime \prime}=2 y^{3}+a_{1} y .
$$

Adapting Whitham's result for the corresponding solution in terms of sech $t$, we find that

$$
a_{1}=\phi^{2}\left(1+12 \sum_{1}^{\infty} \frac{\cosh m \omega_{1} \phi}{\sinh ^{2} m \omega_{1} \phi}\right)>0,
$$

where $\phi=\pi / 2 \beta$.

For the second form we take $g(t)=t^{-1}-\left[t+\omega_{1}(1+i \lambda) / 2\right]^{-1}$ with periods $\omega_{1}$ real and $\omega_{2}=i \lambda \omega_{1}$, which gives

$$
\begin{aligned}
& \psi\left(t ; \omega_{1}\right)=\theta\left(\cot (\theta t)+\tan \left(\theta\left(t+\frac{i \lambda \omega_{1}}{2}\right)\right)\right), \quad \theta=\frac{\pi}{\omega_{1}} \\
& \psi\left(t ; \omega_{2}\right)=\phi\left(\operatorname{coth}(\phi t)-\tanh \left(t+\frac{\omega_{1}}{2}\right)\right), \quad \phi=\frac{\pi}{\lambda \omega_{1}}
\end{aligned}
$$


and the double sums

$$
\begin{array}{r}
G\left(t ; \omega_{2}, \omega_{1}\right)=\phi \sum_{-\infty}^{\infty} \operatorname{coth}\left(\phi t+m \omega_{1}\right)-\tanh \left(\phi\left(t+\left(m+\frac{1}{2}\right) \omega_{1}\right)\right) \\
=\theta\left(\cot \theta t+\sum_{1}^{\infty} \frac{\sin 2 \theta t}{\sin ^{2}(\theta t)+\sinh ^{2}(m \lambda \pi)}\right. \\
\left.\quad+\frac{\sin 2 \theta t}{\cos ^{2}(\theta t)+\sinh ^{2}\left(m-\frac{1}{2}\right) \lambda \pi}\right)
\end{array}
$$

The function $\psi\left(t ; \omega_{2}\right)$ satisfies the differential equation

$$
\psi^{\prime \prime}=2 \psi^{3}+6 \phi \tanh \left(\frac{\pi}{2 \lambda}\right) \psi^{2}+4 \phi^{2} \psi
$$

which reduces to

$$
Y^{\prime \prime}=2 Y^{3}+\phi^{2}\left(4-6 \tanh ^{2}\left(\frac{\pi}{2 \lambda}\right)\left(Y-4 \phi^{3} \tanh \left(\frac{\pi}{2 \lambda}\right) \operatorname{sech}^{2}\left(\frac{\pi i}{2 \lambda}\right)\right)\right),
$$

where $Y=\psi+\phi \tanh (\pi / 2 \lambda)$. Since $4-6 \tanh ^{2}(\pi / 2 \lambda)$ ranges from -2 to 4 , we see that this equation encompasses all three standard forms associated with $a_{3}>0$. Also $\psi, \psi^{\prime} \rightarrow 0$ as $t \rightarrow \pm \infty$, so that these ftunctions represent the separatrices. Apart from the equation represented in standard form by $Y^{\prime \prime}=2 Y^{3}$ $+Y$, this second form provides all the solutions for which $y^{\prime} \neq 0$. In the exceptional case both families coexist, the separating curve being given by the function $y=\theta \cot (\theta t)$ (with $\theta=1 / \sqrt{2}$ ), which represents the limit of each family as the imaginary period tends to infinity.

\section{Conclusion}

For differential equations of the form $y^{\prime \prime}=p(y)$, where $p$ is a polynomial of degree 2 or 3 in $y$, almost all solutions can be represented as the superposition of solutions of simpler equations. In a limited number of cases this is achieved by superposing solutions of the same form, but more commonly the solution represents an alternation of two distinct forms which are themselves solutions of the same equation.

These solutions correspond to elliptic functions whose period parallelogram is a rhombus whose diagonals are parallel to the co-ordinate axes, as distinct from the solutions previously considered, which correspond to elliptic functions with rectangular period parallelograms. 
Most of these new families of solutions are of limited physical interest since they have real periodic singularities, but included in this category are the bulk of the finite periodic solutions for the cubic case when $a_{3}<0$.

\section{References}

E. Goursat (1916), A course in mathematical analysis, Vol. II, Part 1, Functions of a complex variable (Ginn and Company, Boston).

M. Hancock (1910), Lectures on the theory of elliptic functions (Wiley, New York).

G. B. Whitham (1984), 'Comments on periodic waves and solitons', IMA Journal of Applied Mathematics 32, 353-366.

A. A. Zaitsev (1983), 'Formation of stationary non-linear waves by superposition of solitons', Soviet Phys. Dokl. 28(9), 720-722.

Department of Mathematics

University of Queensland

St. Lucia 4067

Australia 